(C) IEEE. Personal use of this material is permitted. However, permission to reprint/republish this material for advertising or promotional purposes or for creating new collective works for resale or redistribution to servers or lists, or to reuse any copyrighted component of this work in other works must be obtained from the IEEE.

This material is presented to ensure timely dissemination of scholarly and technical work. Copyright and all rights therein are retained by authors or by other copyright holders. All persons copying this information are expected to adhere to the terms and constraints invoked by each author's copyright. In most cases, these works may not be reposted without the explicit permission of the copyright holder. 


\title{
STATISTICAL ANALYSIS OF THE IMPACT OF DISTORTION (CORRECTION) ON AN AUTOMATED CLASSIFICATION OF CELIAC DISEASE
}

\author{
M. Liedlgruber ${ }^{1}$, A. Uhl ${ }^{1}$, A. Vécsei ${ }^{2}$ \\ ${ }^{1}$ Department of Computer Sciences, Salzburg University, Austria \\ ${ }^{2}$ Endoscopy Unit, St. Anna Children's Hospital, Vienna, Austria
}

\begin{abstract}
In this work we investigate the impact of barrel-type distortions and distortion correction on an automated classification of endoscopic imagery. For this purpose we use a set of methods from earlier work along with the nearest neighbor classifier on endoscopic images which are distorted in a barrel-type fashion. In addition we classify images after applying distortion correction to them.

Depending on the distance of a patch to the center of distortion a patch is more or less distorted. Hence, we analyze the impact of this distance on the classification accuracy and show that it has an influence on the classification accuracy.

We further investigate whether there is a relationship between the levels of distortion of a patch and the respective nearest neighbor patch. We show that a patch and the respective nearest neighbor patch rather often exhibit the same amount of distortion. We further show that higher distortion differences also have an impact on the probability of misclassification for a patch. The results indicate that for higher distortion differences a patch is more likely to be misclassified.
\end{abstract}

Index Terms - Celiac disease, barrel-type distortion, distortion correction, medical image classification

\section{INTRODUCTION}

Today, medical endoscopy is a widely used procedure to inspect the inner cavities of the human body. As a consequence different medical fields exist for which automated decision-support systems based on endoscopic imagery have been proposed [1]. Since images taken with traditional endoscopes often suffer from various kinds of degradations, a pre-processing of the imagery is often necessary [2] (to cope with e.g. sensor noise, focus and motion blur, and specular reflections [1]).

A different type of degradation, present in all endoscopic images, is a barrel-type distortion. This type of degradation is caused by the wide-angle (fish eye) nature of the optics used in endoscopes (although the strength of the distortion varies depending on the endoscope used). Such a distortion is also claimed to affect diagnosis since it introduces non-linear changes in the image, due to which the outer areas of the image look significant smaller than they actually are [3]. Due to an inhomogeneous magnification such distortions are also suspected to lead to corrupted features [4]. Since the seminal work on distortion correction for endoscopic images [4] several distortion correction procedures have been developed for this application domain $[5,6,7]$.

Corresponding author e-mail: $\{$ mliedl, uhl $\} @$ cosy.sbg.ac.at

This project is partially supported by the Austrian National Bank "Jubiläumsfond", project no. 12991
In a recent study the impact of distortion correction on the classification accuracy regarding celiac disease images has been investigated [8]. Gschwandtner et al. showed that most feature extraction methods evaluated failed to take advantage of applying distortion correction as a pre-processing step to the endoscopic images, resulting in a decreased classification accuracy. To the best of our knowledge, besides the study presented in [8], the potential impact of distortion correction on the analysis of mucosal texture, specifically on the accuracy of corresponding classification techniques, has not been addressed so far.

The focus of the work presented in [8] was an experimental assessment of the impact of distortion correction on the automated classification of celiac disease. This work, in contrast, investigates a potential impact of barrel-type distortions and distortion correction on the automated classification of endoscopic images in the course of a statistical analysis. For this purpose we analyze the impact of the distance of an image patch to the center of distortion $(\mathrm{CoD})$ on the classification accuracy using the nearest neighbor (NN) classifier. We further investigate whether there is a relationship between the distortion of a patch and the distortion of its closest patch (in the sense of the NN classifier). Finally, we study the effect of the distortion difference between a patch and its closest patch on the probability of misclassification of the respective patch.

The remaining part of this work is structured as follows: In Section 2 we describe the background of celiac disease and the staging system commonly used to diagnose this disorder. After a discussion of barrel-type distortions and the problems inherent to this type of degradations in Section 3, we describe the feature extraction methods the results in this work are based on in Section 4. A thorough analysis of experiments carried out and the respective analysis results are given in Section 5. In Section 6 we conclude this work.

\section{CELIAC DISEASE}

Celiac disease, commonly known as gluten intolerance, is a complex autoimmune disorder that affects the small bowel in genetically predisposed individuals of all age groups after introduction of food containing gluten. Characteristic for the disease is an inflammatory reaction in the mucosa of the small intestine. During the course of the disease the mucosa looses its absorptive villi and hyperplasia of the enteric crypts occurs, leading to a diminished ability to absorb nutrients.

Endoscopy with biopsy is currently considered the gold standard for the diagnosis of celiac disease. During standard upper endoscopy at least four duodenal biopsies are taken. Microscopic changes within these specimen are then classified in a histological analysis according to the Marsh classification proposed in 1992 [9]. Subsequently, Oberhuber et al. proposed the modified Marsh 


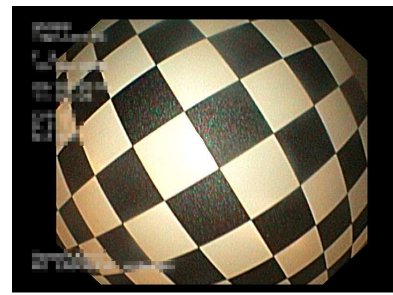

(a)

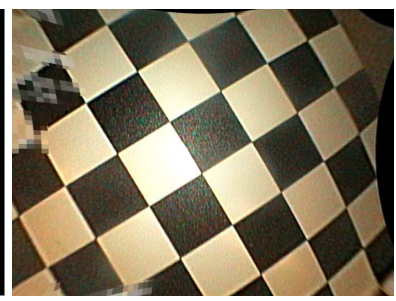

(b)

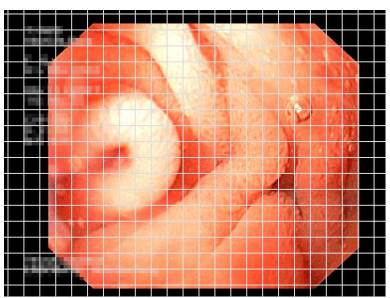

(a)

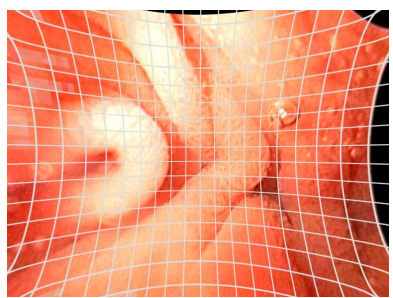

(b)
Fig. 1. Distortion correction. (a) Calibration pattern taken with an Olympus GIF-Q165 endoscope and (b) the respective distortion corrected image.

classification [10] which distinguishes between classes Marsh-0 to Marsh-3, with subclasses Marsh-3a, Marsh-3b, and Marsh-3c, resulting in a total number of six classes. According to the modified Marsh classification Marsh-0 denotes a healthy mucosa (without visible changes of the villous structure) and Marsh-3c designates a complete absence of villi (villous atrophy).

In accordance to the work in [8], we consider the four classes Marsh-0 and Marsh-3a to Marsh-3c only throughout this work, since visible changes in the villi structure can be observed only for classes Marsh-3a to Marsh-3c.

\section{BARREL-TYPE DISTORTIONS AND CORRECTION}

As already mentioned in Section 1, the strength of barrel-type distortions varies depending on the field-of-view (FOV). While a small FOV results in almost no distortions, a high FOV results in a noticeable amount of distortion. In endoscopes FOV values typically range from $100^{\circ}$ to $170^{\circ}$. But the level of distortion is also dependent on the focal length of the optics used. Since endoscopes typically have rather small focal lengths (a few millimeters only), barrel-type distortions get even more amplified. Hence, depending on the FOV and the focal length, barrel-type distortions may vary significantly in strength between the $\mathrm{CoD}$ (which corresponds to the optical axis) and the outer regions of an image (image magnification decreases with the distance to the $\mathrm{CoD}$ ).

To obtain distortion corrected images we employ the OpenCV software developed by J.-Y. Bouguet (a MATLAB version including extensive documentation and examples is also available ${ }^{1}$ ). This software is mainly based on the work of Zhang [11].

For the distortion calibration we use a planar checkerboard pattern (with points on a known grid). The calibration points have been extracted manually since we did not want to loose correction precision due to incorrectly determined corner points. We extracted 140 calibration points out of 4 images for each of the GIF-Q165 endoscopes, which were then fed into the software and applied to our images. Fig. 1 shows an example image of a distorted calibration pattern and the respective image after applying distortion correction. Figure 2 shows the result of applying distortion correction to an endoscopic image (with a grid overlaid to it).

Nevertheless, the distortion correction has the negative sideeffect of interpolation artifacts which are especially apparent in the corners of the image. These artifacts are the result of upsampling and are especially noticeable in the corner regions since, in distorted images, those appear smaller than they actually are. An example for such artifacts is shown in Figure 3.

\footnotetext{
${ }^{1}$ Available at http://www.vision.caltech.edu/bouguetj/calib_doc/
}

Fig. 2. Correction of a barrel-type distortion. (a) Barrel-type distorted endoscopic image (taken with an Olympus GIF-Q165 endoscope with a grid overlaid to the image and (b) the same image after applying distortion correction.

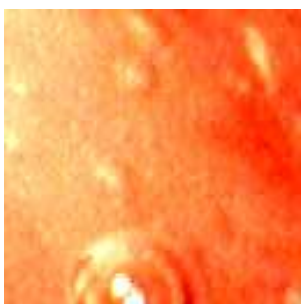

(a)

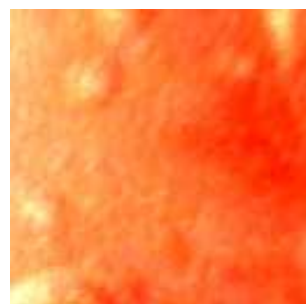

(b)
Fig. 3. Interpolation artifacts due to distortion correction. (a) An example region from a distorted endoscopic image and (b) the same region after applying distortion correction.

\section{TEXTURE CLASSIFICATION}

In order to assess the impact of barrel-type distortion on the classification performance of endoscopic images we use different feature extraction methods to obtain features, which are subsequently classified.

\subsection{Feature extraction}

To obtain features we employ a set of different feature extraction methods based on the frequency domain, which we already used successfully in earlier work for the classification of endoscopic imagery.

This method transforms an image to the wavelet domain using the discrete wavelet transform (maximum number of allowed decomposition levels is set to 3 ). From the most informative subset of subbands (according to a cost function) statistical features are extracted from the respective coefficients [12].

\section{WT-BBC}

The Best Basis Centroids method employs the Best-basis algorithm to find an optimal basis for each image in a training set and computes a centroid over all resulting wavelet packets decomposition structures (maximum number of allowed decomposition levels is set to 4). After transforming all images into this basis, the most informative subset of the resulting subbands (with respect to a cost function) is used to compute the energy over all coefficients within a subband [12].

\section{WT-LDB}

The Local Discriminant Basis algorithm is employed to find an optimal wavelet packet decomposition (maximum number

\section{WT-DWT}


of allowed decomposition levels is set to 4) with respect to the discriminant power between the image classes. After transforming all images into the basis found we use either the coefficient energy or the coefficient variance within a subband as a feature. But instead of using all subbands we limit the number of possible subbands for feature extraction, which allows to control the length of the resulting feature vectors [12].

\section{FFT-Evolved}

By using the FFT an image is transformed into the respective power spectrum. Multiple ring-shaped filters are then applied to the spectra to concentrate on discriminative frequency subbands only. Since the number of possible rings is quite large, an evolutionary algorithm is used to find an optimal set of filters for each color channel. For each of the resulting subbands the mean of the coefficient magnitudes is used as a feature [13].

\section{WT-GMRF}

In this method an image is first transformed to the wavelet domain using the discrete wavelet transform (maximum number of allowed decomposition levels is set to 3). For each of the resulting detail subbands Gaussian Markov Random field parameters are estimated (using Geman type neighborhoods). In addition to the Markov parameters this method also uses the approximation error for each subband as a feature. [14]

\subsection{Classification}

To classify the features we use the nearest neighbor (1-NN) classifier in conjunction with the Euclidean distance. We use this rather simple classifier since in this work we are not interested in achieving the best possible classification rates. Instead we are only interested in the impact of distortion (correction) on the classification rates. While for other classifiers the results most likely will differ, the general impact of distortion (correction) on the classification rates is expected to be similar since the distortion (correction) affects the features extracted.

The parameters for the different methods have been chosen based on experiences gained from earlier work.

\section{EXPERIMENTS AND ANALYSIS}

\subsection{Experimental Setup}

The images used throughout this work have been taken during duodenoscopies at the St. Anna Children's Hospital using gastroscopes without magnification (two Olympus GIF-Q165 endoscopes with an image resolution of $768 \times 576$ pixels, a FOV of $140^{\circ}$, and slightly different CoDs). The slightly different CoDs are due to marginal differences between the optics used in the endoscopes. However, the distance between the two CoDs is minimal with about 4 pixels only.

The main indications for endoscopy were the diagnostic evaluation of dyspeptic symptoms, positive celiac serology, anemia, malabsorption syndromes, inflammatory bowel disease, and gastrointestinal bleeding. Images have been recorded using the modified immersion technique, which is based on the instillation of water into the duodenal lumen for better visibility of the villi. The tip of the gastroscope is inserted into the water and images of interesting areas are taken. A study [15] shows that the visualization of villi with the immersion technique has a higher positive predictive value. Previous work [16] also found that the modified immersion technique is more suitable for automated classification purposes as compared to the classical image capturing technique.

\begin{tabular}{lcccc}
\hline & Marsh-0 & \multicolumn{3}{c}{ Marsh-3 } \\
\hline Bulbus & 125 & & 111 & \\
Pars & 97 & & 159 & \\
\hline \hline & Marsh-0 & Marsh-3a & Marsh-3b & Marsh-3c \\
\hline Bulbus & 125 & 45 & 48 & 18 \\
Pars & 97 & 42 & 53 & 64 \\
\hline
\end{tabular}

Table 1. Distribution of the patches used throughout this work in the two-classes case and the four-classes case.

We created two sets of textured image patches with optimal quality to analyze the effect of of barrel-type distortions under "idealistic" conditions. Thus, the captured data was inspected and filtered by several qualitative factors (sharpness, distortions, and visibility of features). In the next step, texture patches with a fixed size of $128 \times 128$ pixels were extracted (a size which turned out to be optimally suited in earlier experiments on automated celiac disease diagnosis [16]). The images have been captured in two duodenal regions, i.e. the Duodenal Bulb (Bulbus duodeni) and the Pars Descendens (abbreviated in the rest of the paper as Bulbus and Pars). The sets of patches have been extracted from 63 and 76 patients in case of the Bulbus set and the Pars set, respectively.

In order to generate ground truth for the texture patches used in experimentation, the condition of the mucosal areas covered by the images was determined by histological examination of biopsies from the corresponding regions. Severity of villous atrophy was classified according to the modified Marsh classification.

The number of extracted images is 236 and 256 in case of the Bulbus and Pars region, respectively. The detailed ground truth is shown in Table 1. While in the two-classes case the patches are balanced rather well, in the four-classes case the Marsh-3 classes contain less images as compared to Marsh-0. As a consequence we are not able to split the image sets into separate training and validation sets. To be able to produce reliable result estimates we therefore use Leave-One-Out cross validation (LOO-CV). In this scenario one image out of an image set is considered to be an unknown sample which is classified using a classifier trained with the remaining images. This process is repeated for each single image in the image database.

Prior to the actual classification we pre-process each image by applying CLAHE [17] followed by a Laplace Sharpening with a kernel size of $9 \times 9$ [18] since this combination of pre-processing steps turned out to be optimal in earlier work.

Since after distortion correction these data do no longer correspond to squares - as can be noticed from Figure 2 - these cannot be used immediately for subsequent classification (the techniques used implicitly assume at least a rectangularly shaped texture patch). To overcome this problem, in case of distortion correction, we compute the new position of a patch according to the underlying distortion and extract a new $128 \times 128$ pixels patch at the newly computed location.

\subsection{Classification results}

Prior to the actual CoD analysis results we briefly present the classification accuracies which have been obtained using the image databases according to the ground truth shown in Table 1 .

We immediately notice that, similar to the results published in [8], the results for the distortion corrected images are constantly lower compared to the results obtained for the distorted images (for all methods, in the two-classes case as well as in the four-classes 


\begin{tabular}{lcccc}
\hline & Cls & Dist $\left(\mathbf{R}_{\mathbf{1}}\right)$ & $\mathbf{D C}\left(\mathbf{R}_{\mathbf{2}}\right)$ & $\mathbf{R}_{\mathbf{2}}-\mathbf{R}_{\mathbf{1}}$ \\
\hline \multirow{5}{*}{ WT-DWT } & 2 & $86.4 \%$ & $80.1 \%$ & $-6.3 \%$ \\
& 4 & $70.3 \%$ & $62.3 \%$ & $-8.0 \%$ \\
\hline \multirow{2}{*}{ WT-BBCB } & 2 & $89.8 \%$ & $80.5 \%$ & $-9.3 \%$ \\
& 4 & $70.8 \%$ & $69.9 \%$ & $-0.9 \%$ \\
\hline \multirow{2}{*}{ WT-LDB } & 2 & $91.9 \%$ & $77.5 \%$ & $-14.4 \%$ \\
& 4 & $72.9 \%$ & $66.1 \%$ & $-6.8 \%$ \\
\hline \multirow{2}{*}{ WT-GMRF } & 2 & $91.5 \%$ & $86.9 \%$ & $-4.6 \%$ \\
& 4 & $75.4 \%$ & $63.1 \%$ & $-12.3 \%$ \\
\hline \multirow{2}{*}{ FFT-Evolved } & 2 & $97.0 \%$ & $94.1 \%$ & $-2.9 \%$ \\
& 4 & $83.5 \%$ & $82.6 \%$ & $-0.9 \%$ \\
\hline \multirow{2}{*}{ WT-DWT } & 2 & $72.7 \%$ & $65.2 \%$ & $-7.5 \%$ \\
& 4 & $43.4 \%$ & $39.5 \%$ & $-3.9 \%$ \\
\multirow{2}{*}{ WT-BBCB } & 2 & $69.9 \%$ & $65.2 \%$ & $-4.7 \%$ \\
& 4 & $48.4 \%$ & $46.9 \%$ & $-1.5 \%$ \\
\hline \multirow{2}{*}{ WT-LDB } & 2 & $74.2 \%$ & $66.0 \%$ & $-8.2 \%$ \\
& 4 & $52.0 \%$ & $41.4 \%$ & $-10.6 \%$ \\
\hline \multirow{2}{*}{ WT-GMRF } & 2 & $74.2 \%$ & $71.9 \%$ & $-2.3 \%$ \\
& 4 & $49.6 \%$ & $45.7 \%$ & $-3.9 \%$ \\
\hline \multirow{2}{*}{ FFT-Evolved } & 2 & $86.7 \%$ & $84.0 \%$ & $-2.7 \%$ \\
& 4 & $64.1 \%$ & $63.3 \%$ & $-0.8 \%$ \\
\hline
\end{tabular}

Table 2. Comparison of the overall classification accuracies between the distorted and the distortion corrected (DC) image sets.

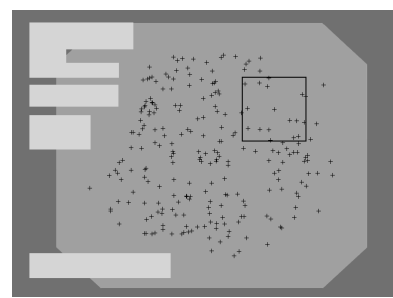

(a) Bulbus set

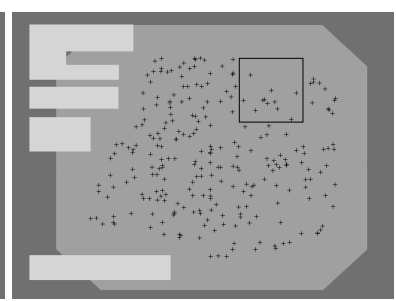

(b) Pars set
Fig. 4. Distribution of the centers of the distorted patches extracted (along with one example patch). The light gray blocks in the upper and lower left corner denote the approximate areas covered by patient information.

case). The method suffering the least from the distortion correction is FFT-Evolved, which is due to the feature selection (evolutionary algorithm) used by this method.

\subsection{Analysis Results}

In case only patches equidistant to the $\mathrm{CoD}$ would be employed in classification, an analysis regarding the $\mathrm{CoD}$ distances and the according levels of distortion of the patches would be meaningless. This is due the roughly equal level of distortion the patches would exhibit in such a case. In order to clarify this we display the center position of all image patches used in Figure 4 (the distorted patches) and Figure 5 (undistorted patches).

We notice that the patches are well distributed (except for the areas where patient related information is overlaid in the upper and lower left corner). Moreover, the distribution is sufficient to perform a CoD-related analysis since this figure also shows that it is not the case that the sets contain patches close to the CoD only.

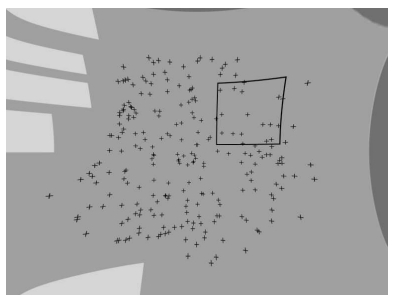

(a) Bulbus set

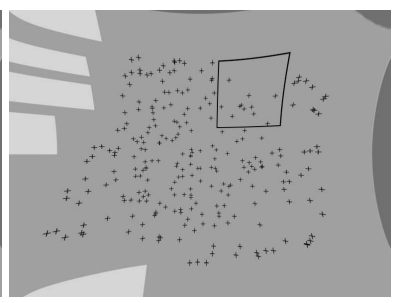

(b) Pars set
Fig. 5. Distribution of the patch centers after applying distortion correction (along with the distorted example patch).

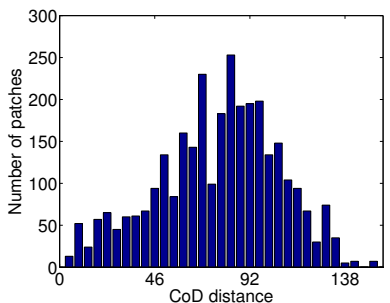

(a)

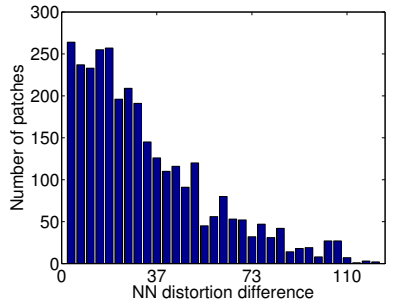

(c)

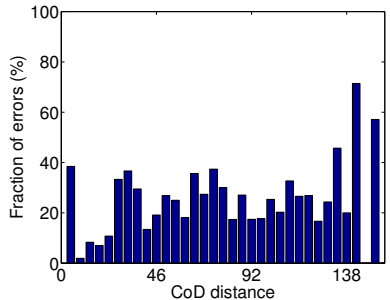

(b)

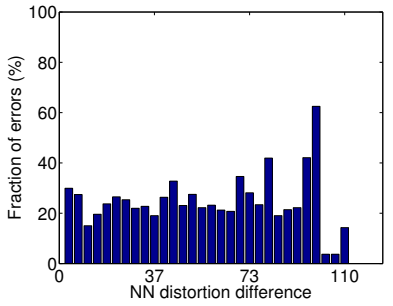

(d)
Fig. 6. CoD analysis (distorted patches, additive mode).

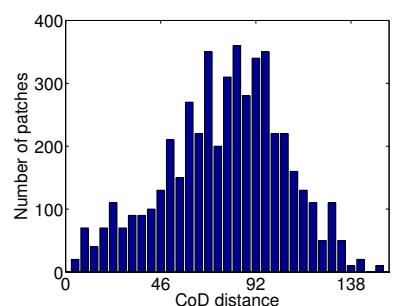

(a)

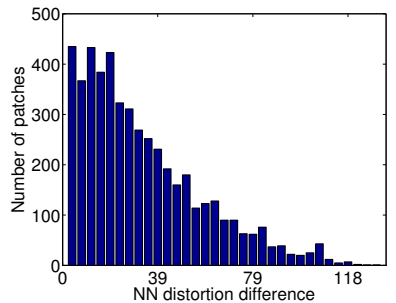

(c)

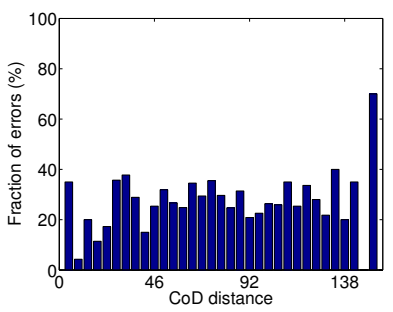

(b)

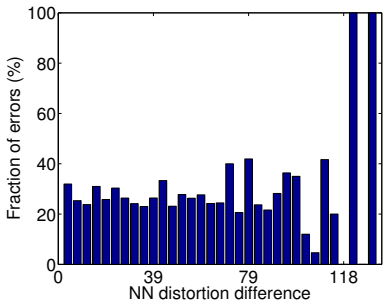

(d)
Fig. 7. CoD analysis (distorted patches, mixed mode). 


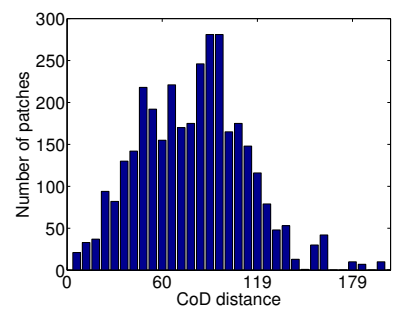

(a)

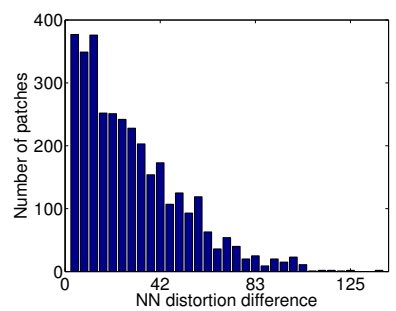

(c)

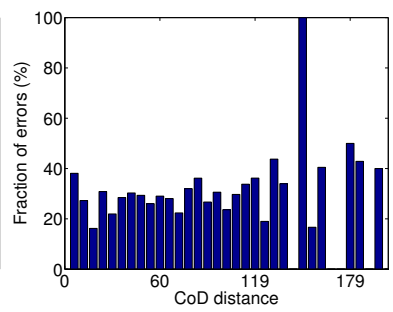

(b)

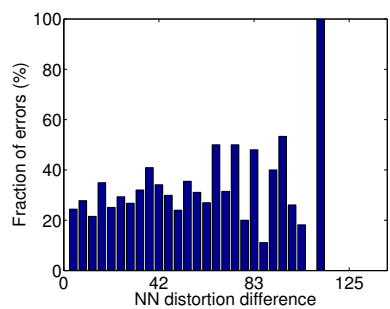

(d)
Fig. 8. CoD analysis (undistorted patches, additive mode).

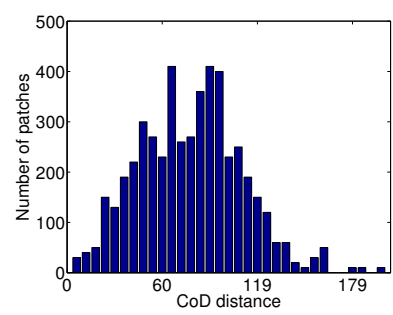

(a)

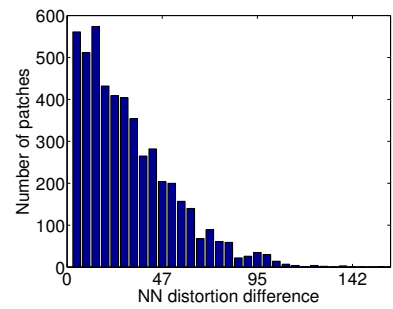

(c)

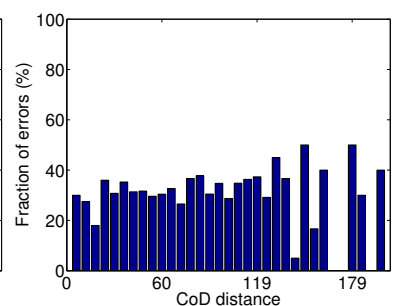

(b)

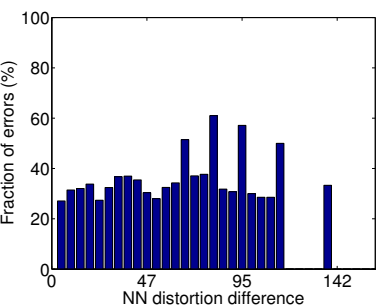

(d)
Fig. 9. $\mathrm{CoD}$ analysis (undistorted patches, mixed mode)

\subsubsection{Analysis modes}

Since the endoscopes used to acquire the image sets exhibit slightly different CoDs (we refer to these endoscopes as G1 and G2), we chose to implement two distinct analysis modes to generate our plots.

In the first mode a patch and his closest patch (in the sense of $\mathrm{NN}$ classification) must have been taken with the same endoscope (either G1 or G2). This results in one histogram for each endoscope, which, after adjusting the histogram ranges to be equal, are accumulated to obtain one final histogram. This analysis mode is referred to as "additive mode". In the second mode the restriction on a single endoscope is lifted (we call this mode "mixed mode"). Hence, a single histogram, based on more patches, is the result.
No matter which mode we use, the patches from the Bulbus set and the Pars set are combined, which is eligible since the same endoscopes have been used.

\subsubsection{Effect of CoD distance on classification}

Figures 6(a), 7(a), 8(a), and 9(a) show the distribution of the distances of the patches used to the CoDs. As we notice from these figures, the distributions are roughly equal no matter whether we use distorted or undistorted patches. The difference between the different analysis modes is also only marginal.

Figures 6(b), 7(b), 8(b), and 9(b) show the fraction of misclassified patches depending on the respective $\mathrm{CoD}$ distances. To obtain these figures we looked up the closest patch $N N\left(P_{i}\right)$ for each patch $P_{i}$ (in the sense of the nearest neighbor classifier). If the image class of patch $P_{i}$ does not coincide with the class of patch $N N\left(P_{i}\right)$ we increment the bin in the histogram which corresponds to the CoD distance of patch $P_{i}$.

While for lower CoD distances the fraction of misclassified patches is not dependent on the CoD distance, this changes for higher distances. From these figures it seems that there is indeed a trend to misclassification for higher $\mathrm{CoD}$ distance values. In case of the distorted images this can be explained by the barrel-type distortion which is especially noticeable in the outer areas of an image. When applying distortion correction, again areas further away from the CoD get more distorted due to the interpolation involved into the distortion correction.

\subsubsection{Distortion difference between neighboring patches}

Figures 6(c), 7(c), 8(c), and 9(c) show the distribution of the distortion difference between a patch $P_{i}$ and his closest patch $N N\left(P_{i}\right)$. In the distorted case as well as in the undistorted case the distance of a patch to the $\operatorname{CoD} D\left(P_{i}, C O D\right)$ also reflects the degree of distortion of the patch - in the distorted case due to the barrel-type distortion and in the undistorted case due to the interpolation artifacts generated. Hence, the difference $\left|D\left(P_{i}, C O D\right)-D\left(N N\left(P_{i}\right), C O D\right)\right|$ can be regarded as the difference of distortion between the two patches.

As we notice from figures 6(c), 7(c), 8(c), and 9(c) the distortion difference between a patch $P_{i}$ and $N N\left(P_{i}\right)$ is in most cases rather low. In other words, $N N\left(P_{i}\right)$ is in most cases chosen such that the level of distortion is roughly equal between $P_{i}$ and $N N\left(P_{i}\right)$. This is not surprising since the closest patch is chosen based on the features extracted from the patches. The methods we used for the analysis are based on the underlying textural content which however is affected by distortions. As already pointed out above, corrupted texture appears in case of barrel-type distortions (distorted case) as well as in the case of distortion correction (due to the interpolation artifacts). Hence, this trend can be observed in case of distorted images as well as in the undistorted case (for both analysis modes).

Figures 6(d), 7(d), 8(d), and 9(d) show that the fraction of misclassified patches depends on the distortion difference between $P_{i}$ and $N N\left(P_{i}\right)$. As a consequence, a patch farther away (in the radial sense) from its closest patch (in terms of the NN classifier) is more likely to be misclassified. Similar to the figures 6(b), 7(b), 8(b), and 9(b) we notice that for small distortion differences no clear tendency can be observed. However, for large distortion differences the probability of misclassification is indeed higher. This trend is noticeable for the distorted patches as well as for the undistorted patches. Again, this phenomenon is linked to either barrel-type distortions or interpolation artifacts in case of distortion correction. 


\section{CONCLUSION}

In this work we investigated the impact of barrel-type distortions and distortion corrections on the classification accuracy of certain methods used in earlier work. We have shown that in case of distortion as well as after distortion correction the fraction of misclassified patches gets higher for patches with greater distances to the $\mathrm{CoD}$. We have also shown that the higher the distortion difference between a patch and his closest patch the more likely a patch gets misclassified.

Considering the fact that the features used are rather sensitive to distortions this outcome is not surprising at all, since, no matter whether we use distorted images or apply distortion correction, in both cases certain types of distortions are apparent in the outer regions of the images (barrel-type distortions in case of the distorted images and interpolation artifacts in case of distortion correction). This is also confirmed by the fact that in [8] edge-based features have been shown to be more resistant against distortions.

In future work we will therefore explore the impact of barreltype distortions and distortion correction on edge-based features. In addition we will also investigate the parameter-free distortion correction, more recently proposed by Barreto et al [19].

\section{REFERENCES}

[1] M. Liedlgruber and A. Uhl, "Endoscopic image processing an overview," in Proceedings of the 6th International Symposium on Image and Signal Processing and Analysis (ISPA'09), Salzburg, Austria, Sept. 2009, pp. 707-712.

[2] G. Hanna and A. Cuschieri, "Image display technology and image processing," World Journal of Surgery, vol. 25, no. 11, pp. 1419-1427, Nov. 2001.

[3] T. B. Borcharrt, A. Conci, and M. C. d'Ornellas, "A warping based approach to correct distortions in endoscopic images," in Proceedings of the 22nd Brazilian Symposium on Computer Graphics and Image Processing (Sibgrapi'09), Rio de Janeiro, Brazil, Oct. 2009.

[4] H. Haneishi, Y. Yagihashi, and Y. Miyake, "A new method for distortion correction of electronic endoscope images," IEEE Transactions on Medical Imaging, vol. 14, no. 3, pp. 548-55, Sept. 1995.

[5] K. V. Asari, S. Kumar, and D. Radhakrishnan, "A new approach for nonlinear distortion correction in endoscopic images based on least squares estimation," IEEE Transactions on Medical Imaging, vol. 18, no. 4, pp. 345-354, Apr. 1999.

[6] J. P. Helferty, C. Zhang, G. McLennan, and W. E. Higgins, "Videoendoscopic distortion correction and its application to virtual guidance of endoscopy," IEEE Transactions on Medical Imaging, vol. 20, no. 7, pp. 605-617, July 2001.

[7] H.-X. Sun, Y.-H. Zhang, and F.-L. Luo, "A novel approach for nonlinear distortion correction of industrialendoscope images," in Proceedings of the 17th World Conference on Nondestructive Testing, Shanghai, China, Oct. 2008, pp. 25-28.

[8] M. Gschwandtner, M. Liedlgruber, A. Uhl, and A. Vécsei, "Experimental study on the impact of endoscope distortion correction on computer-assisted celiac disease diagnosis," in Proceedings of the 10th International Conference on Information Technology and Applications in Biomedicine (ITAB'10), Corfu, Greece, Nov. 2010.
[9] M. Marsh, "Gluten, major histocompatibility complex, and the small intestine. a molecular and immunobiologic approach to the spectrum of gluten sensitivity ('celiac sprue')," Gastroenterology, vol. 102, no. 1, pp. 330-354, Jan. 1992.

[10] G. Oberhuber, G. Granditsch, and H. Vogelsang, "The histopathology of coeliac disease: time for a standardized report scheme for pathologists," European Journal of Gastroenterology and Hepatology, vol. 11, pp. 1185-1194, Nov. 1999.

[11] Z. Zhang, "A flexible new technique for camera calibration," IEEE Transactions on Pattern Analysis and Machine Intelligence, vol. 22, no. 11, pp. 1330-1334, Nov. 2000.

[12] M. Liedlgruber and A. Uhl, "Statistical and structural wavelet packet features for pit pattern classification in zoomendoscopic colon images," in Proceedings of the 7th WSEAS International Conference on Wavelet Analysis \& Multirate Systems (WAMUS'07), Arcachon, France, Oct. 2007, pp. 147-152.

[13] A. Vécsei, T. Fuhrmann, M. Liedlgruber, L. Brunauer, H. Payer, and A. Uhl, "Automated classification of duodenal imagery in celiac disease using evolved fourier feature vectors," Computer Methods and Programs in Biomedicine, vol. 95, no. 2, pp. S68-S78, Aug. 2009.

[14] M. Häfner, A. Gangl, M. Liedlgruber, A. Uhl, A. Vécsei, and F. Wrba, "Combining Gaussian Markov random fields with the discrete wavelet transform for endoscopic image classification," in Proceedings of the 17th International Conference on Digital Signal Processing (DSP'09), Santorini, Greece, July 2009, pp. 177-182.

[15] A. Gasbarrini, V. Ojetti, L. Cuoco, G. Cammarota, A. Migneco, A. Armuzzi, P. Pola, and G. Gasbarrini, "Lack of endoscopic visualization of intestinal villi with the immersion technique in overt atrophic celiac disease," Gastrointestinal endoscopy, vol. 57, pp. 348-351, Mar. 2003.

[16] S. Hegenbart, R. Kwitt, M. Liedlgruber, A. Uhl, and A. Vécsei, "Impact of duodenal image capturing techniques and duodenal regions on the performance of automated diagnosis of celiac disease," in Proceedings of the 6th International Symposium on Image and Signal Processing and Analysis (ISPA'09), Salzburg, Austria, Sept. 2009, pp. 718-723.

[17] K. Zuiderveld, "Contrast limited adaptive histogram equalization," in Graphics Gems IV, Paul S. Heckbert, Ed., pp. 474485. Morgan Kaufmann, 1994.

[18] R. C. Gonzalez and R. E. Woods, Digital Image Processing, Prentice-Hall, 2nd edition, 2002.

[19] J. P. Barreto, R. Swaminathan, and J. Roquette, "Non parametric distortion correction in endoscopic medical images," in Proceedings of the 3DTV Conference 2007, Kos, Greece, May 2007, pp. 1-4. 